\title{
Miscible displacements in Hele-Shaw cells: Nonmonotonic viscosity profiles
}

\author{
D. Schafroth, N. Goyal, E. Meiburg* \\ Department of Mechanical Engineering, University of California, Santa Barbara, CA 93106, USA
}

Received 1 May 2006; received in revised form 23 August 2006; accepted 11 September 2006

Available online 6 October 2006

\begin{abstract}
The influence of nonmonotonic viscosity-concentration relationships on viscous fingering of neutrally buoyant, miscible fluids in a Hele-Shaw cell has been investigated. In a first step, quasisteady base states are obtained by means of nonlinear Stokes simulations. The properties of these base states are analyzed as a function of the Péclet number, the viscosity ratio, and the profile parameters. Subsequently, the stability of these base states is investigated by means of a linear stability analysis. Overall, the nonmonotonicity of the viscosity-concentration relationship is seen to have a much smaller influence on Hele-Shaw displacements than on corresponding Darcy flows. The reason for this difference lies in the nature of the respective base states. For Darcy flows, the base state is characterized by constant velocity and a diffusively decaying concentration (and hence viscosity) profile. This base viscosity profile is strongly affected by the nonmonotonicity. On the other hand, for Hele-Shaw displacements the quasisteady base states are convectively dominated and characterized by sharp fronts, so that their shape depends only weakly on the details of the viscosity-concentration relationship. Hence, for Hele-Shaw displacements both the eigenfunctions and the associated growth rates are quite similar for monotonic and nonmonotonic profiles, in contrast to the findings by [O. Manickam, G.M. Homsy, Stability of miscible displacements in porous media with nonmonotonic viscosity profiles, Phys. Fluids A 5 (1993) 1356-1367] for Darcy flows.
\end{abstract}

(c) 2006 Elsevier Masson SAS. All rights reserved.

Keywords: Hele-Shaw flow; Stokes equations; Miscible displacement; Nonmonotonic viscosity profile; Linear stability analysis

\section{Introduction}

Hele-Shaw cells are frequently employed in order to investigate aspects of porous media displacements, cf. [2]. In addition this geometry, in its own right, is relevant to numerous application areas, from lubrication problems, bearing flows, and oil displacements in fractured rocks, to small-scale MEMS devices. It is well known that the analogy between Hele-Shaw flows and displacements in true porous media is an incomplete one. Hence it is necessary to establish the limits of this analogy carefully, in order to avoid drawing misleading conclusions. Towards this goal, [3] recently compared linear stability results for miscible Hele-Shaw displacements governed by the Stokes equations with corresponding Darcy results for porous media. As a first step, the authors establish the quasisteady base states of

\footnotetext{
* Corresponding author.

E-mail address: meiburg@engineering.ucsb.edu (E. Meiburg).
} 
two-dimensional Hele-Shaw displacements by means of nonlinear Stokes simulations. They find the front thickness of this quasisteady state to scale with $\mathrm{Pe}^{-1 / 2}$, while it depends only weakly on the viscosity ratio. In a subsequent step, the stability of the quasisteady front to spanwise perturbations is examined, based on the three-dimensional Stokes equations. Generally poor agreement is observed between the Stokes results and corresponding findings for Darcy flows [4]. [5] extend this investigation to variable density displacements in vertical Hele-Shaw cells and find excellent agreement with the experimental data of [6] regarding the most amplified wavelength. These results establish Stokes based direct simulations and accompanying linear stability analyses as powerful tools for comparing Hele-Shaw displacements with their Darcy based counterparts.

For miscible displacements governed by Darcy's law, [1] and [7] obtained interesting linear stability results and nonlinear dynamics if the two fluids give rise to nonmonotonic viscosity profiles, cf. also the investigation by [8]. In particular, the authors find that even for a favorable (unfavorable) endpoint viscosity contrast the displacement can be unstable (stable). Furthermore, the diffusion of the base state can be destabilizing, so that initially stable nonmonotonic profiles can become unstable after some time. In order to identify the cause for this unexpected behavior, the authors analyze the eigenfunctions of the flow, with a particular focus on the structure of the vorticity and streamfunction fields. They observe a quadrupole structure of the vorticity field, similar to the investigations by [9] for radially dominated displacements with nonmonotonic profiles, and by $[10,11]$ for shear stabilized displacements, which can give rise to dynamics fundamentally different from the dipole structure that dominates the evolution of monotonic displacements [4]. The goal of the present investigation is to establish if such unconventional behavior is limited to Darcy based flows, or if it can be observed in Hele-Shaw displacements as well. As shown by [12,3], Hele-Shaw flows are strongly influenced by the presence of an additional length scale in the form of the gap width of the cell, which affects both the shape of the quasisteady state, as well as its linear stability characteristics.

The present investigation is structured as follows: In a first step, we will establish the structure of the twodimensional base state for nonmonotonic displacements, and compare its features with those of base states for monotonic profiles. Subsequently, the linear stability analysis will be performed for the nonmonotonic base states. A discussion of the findings concludes the paper.

\section{Physical problem}

The investigation focuses on constant density, miscible fluids of different viscosities in a Hele-Shaw cell, as shown in Fig. 1. The less viscous fluid 1 on the left displaces the more viscous fluid 2 to the right. For narrow gap widths $e$, the flow velocities will be small, so that the fluid motion can be described by the three-dimensional Stokes equations

$$
\begin{aligned}
& \nabla \cdot \vec{u}=0, \\
& \nabla p=\nabla \cdot \boldsymbol{\tau}, \\
& \frac{\partial c}{\partial t}+\vec{u} \cdot \nabla c=D \nabla^{2} c .
\end{aligned}
$$

Here $\vec{u}$ denotes the flow velocity, while $c$ indicates the relative concentration of the more viscous fluid. $\tau$ represents the viscous stress tensor for Newtonian fluids, and $D$ refers to the diffusion coefficient, which is assumed constant. $x, y, z$ indicate the spanwise, streamwise and cross-gap directions, respectively.

The viscosity $\mu$ is a function of the concentration $c$ and will depend on the fluid pair used. As [1] point out, there are a variety of fluid combinations for which the viscosity-concentration relationship is nonmonotonic. In order to be able to compare the present Stokes results with their earlier findings for Darcy flows, we employ the same functional

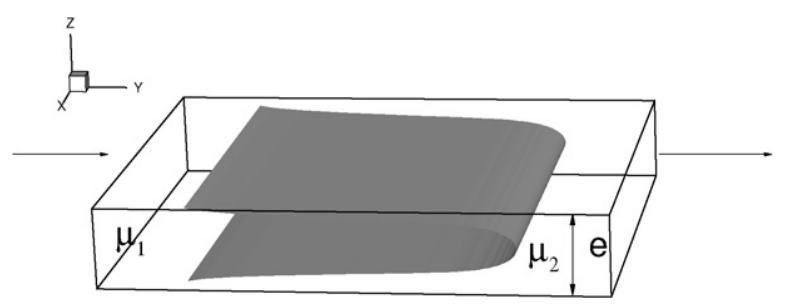

Fig. 1. Miscible displacement in a Hele-Shaw cell. The less viscous fluid on the left displaces the more viscous fluid on the right. 
relationships between viscosity and concentration as [1]. For an endpoint viscosity ratio $\alpha=\mu_{2} / \mu_{1}$, the viscosity profile $\mu(c)$ is given by a sine function modified through a sequence of transformations as follows

$$
\begin{aligned}
& \mu(c)=\mu_{1} \cdot \mu_{m} \sin (\xi), \\
& \xi=\xi_{0}(1-\eta)+\xi_{1} \eta, \quad \eta=\frac{(1+a) c}{1+a c},
\end{aligned}
$$

where

$$
\xi_{0}=\sin ^{-1}\left(\frac{\alpha}{\mu_{m}}\right), \quad \xi_{1}=\pi-\sin ^{-1}\left(\frac{1}{\mu_{m}}\right),
$$

and

$$
a=\frac{\left(1-c_{m}\right)-\eta_{m}}{\left(1-c_{m}\right)\left(\eta_{m}-1\right)}, \quad \eta_{m}=\frac{\pi / 2-\xi_{0}}{\xi_{1}-\xi_{0}} .
$$

The profile has the end-point viscosities $\mu(0)=1$ and $\mu(1)=\alpha$, and a maximum value $\mu_{m}$ at the concentration $c=c_{m}$. In following [1], we introduce the logarithm $R$ of the viscosity ratio defined as

$$
R=\ln \frac{\mu_{2}}{\mu_{1}}=\ln (\alpha),
$$

and a parameter $\Lambda$ that relates the end point slopes of the viscosity profile

$$
\Lambda=\frac{\mathrm{d} \mu /\left.\mathrm{d} c\right|_{c=0}+\mathrm{d} \mu /\left.\mathrm{d} c\right|_{c=1}}{\alpha+1} .
$$

For monotonic profiles $\Lambda$ is always positive, while for nonmonotonic profiles $\Lambda$ depends on the magnitude of the gradient at the end points. $\Lambda<0$ indicates that the slope of the viscosity profile at $c=1$ is steeper than at $c=0$, while $\Lambda>0$ denotes the reverse scenario.

The governing equations are rendered dimensionless by introducing a characteristic length $L^{*}$, velocity $U^{*}$, time $T^{*}$, pressure $P^{*}$ and viscosity $\mu^{*}$ in the form of

$$
\begin{aligned}
L^{*} & =e, \\
U^{*} & =U, \\
T^{*} & =\frac{e}{U}, \\
P^{*} & =\frac{\mu_{1} U}{e}, \\
\mu^{*} & =\mu_{1},
\end{aligned}
$$

where $U$ refers to the average velocity across the gap of the Hele-Shaw cell. We thus obtain the set of dimensionless equations as

$$
\begin{aligned}
& \nabla \cdot \vec{u}=0, \\
& \nabla p=\nabla \cdot \tau, \\
& \frac{\partial c}{\partial t}+\vec{u} \cdot \nabla c=\frac{1}{P e} \nabla^{2} c,
\end{aligned}
$$

where the Péclet number defined as

$$
P e=\frac{U e}{D}
$$

indicates the relative magnitude of convective to diffusive transport of the concentration. 


\section{Stokes flow simulations}

In order to establish the quasisteady base states by means of nonlinear, two-dimensional Stokes flow simulations, we follow the computational strategy described by [3]. The required discretization of 1,025 and 193 points in the $y$ - and $z$-directions was established by means of careful convergence tests. In order to evaluate the influence of the governing dimensionless parameters, we conducted simulations for $P e=500,1,000$ and 2,000; $R=2,3$ and 5; $\mu_{m}=1.1,1.5$ and 2 ; and $\Lambda=-15,-10,-3,0,3,10$ and 15 .

\subsection{Evolution of the quasisteady displacement front}

Upon the start of the flow, the velocity field immediately deforms the concentration distribution in the interior of the cell, while the concentration at the wall changes due to diffusion only. This change in the concentration field, in turn, modifies the viscosity field, thus leading to the formation of a well-defined finger of the less viscous fluid that propagates along the center of the Hele-Shaw cell (Fig. 2). This finger exhibits a steep concentration front at its tip, and diffusively spreading concentration layers along its sides. For sufficiently large values of $P e$ and $R$, the finger tip will reach a quasisteady state in a moving reference frame. The base state for the corresponding monotonic profile with an exponential viscosity-concentration relationship, shown for comparison, demonstrates that the differences in the base state due to nonmonotonicity are minor. For small Péclet numbers, diffusion outweighs convection, so that

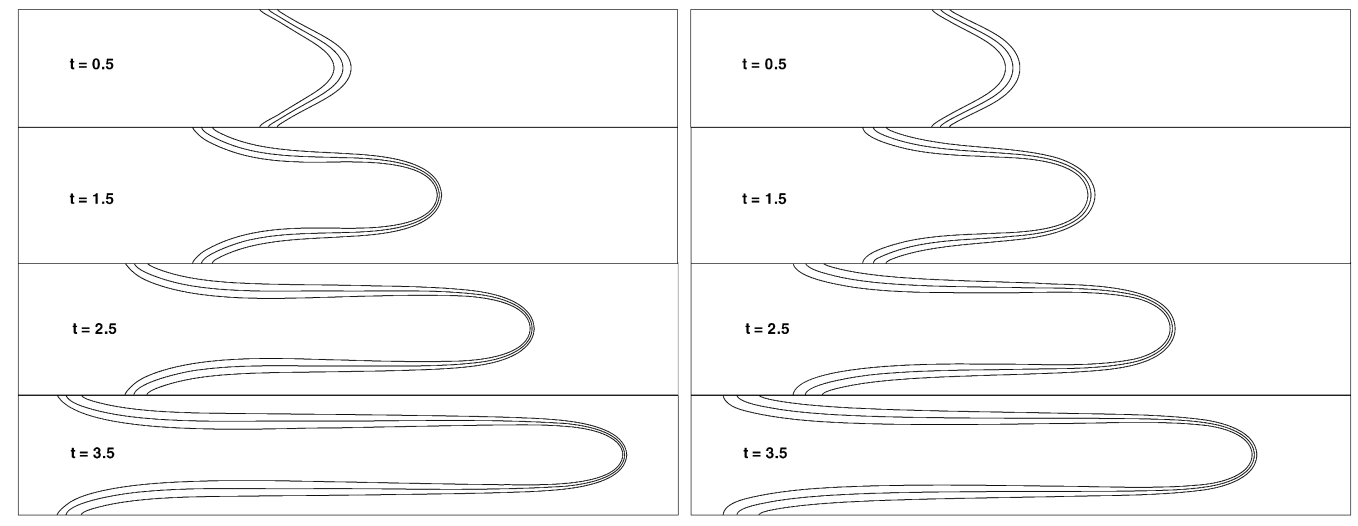

Fig. 2. Evolution of a quasisteady base state near the finger tip for $P e=2,000$ and $R=5$. Left: Monotonic, exponential profile. Right: Nonmonotonic profile with $\Lambda=0$ and $\mu_{m}=2$. The quasisteady base states of the two cases show small differences, but are quantitatively very similar overall.

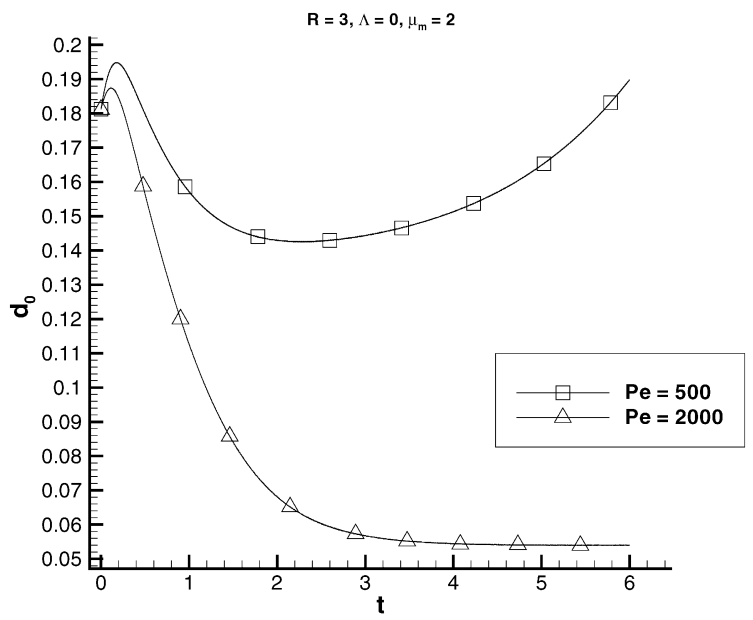

Fig. 3. Evolution of the front thickness $d_{0}$ as a function of time for $P e=500$ and 2,000, respectively $\left(R=3, \Lambda=0, \mu_{m}=2\right)$. While a quasisteady state evolves for the larger $P e$-value, this is not the case for $P e=500$, as diffusive effects prevent the formation of a sharp concentration front at the finger tip. 

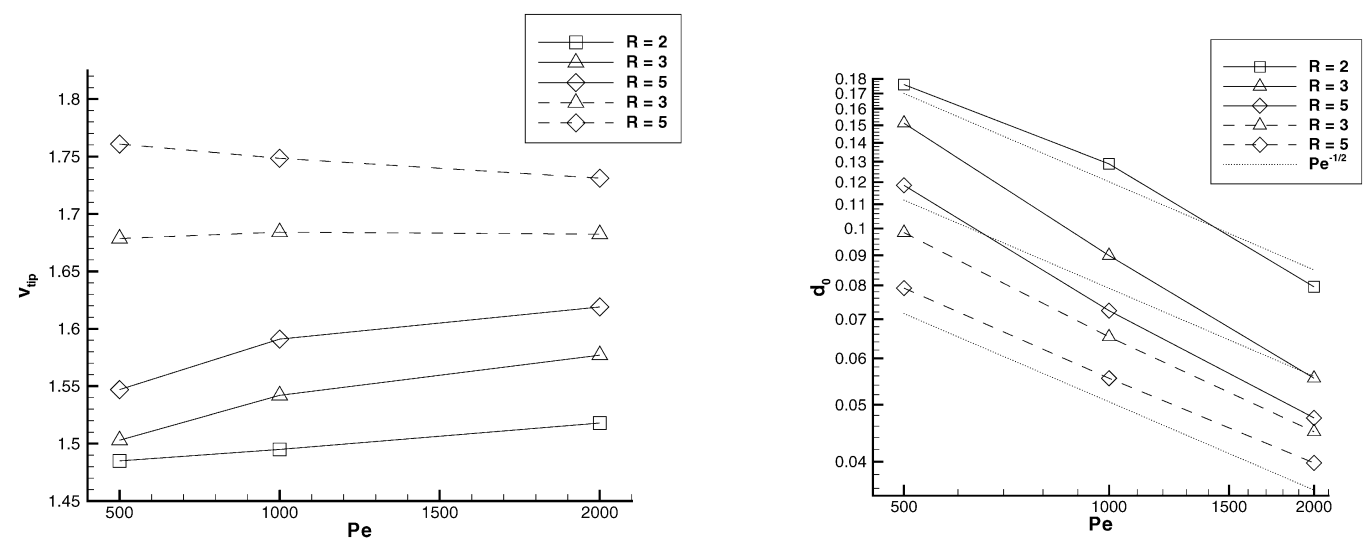

Fig. 4. Tip velocity $v_{\text {tip }}$ (left) and front thickness $d_{0}$ (right) of the quasisteady displacement front as a function of $P e$ for different viscosity ratios. Solid lines: nonmonotonic profiles with $\Lambda=0$ and $\mu_{m}=2$, dashed lines: exponential monotonic profiles. The tip velocity of the quasisteady state is generally lower for nonmonotonic profiles, as compared to their monotonic counterparts. While the monotonic profiles closely follow a $P e^{-1 / 2}$ scaling, this is not the case for the nonmonotonic profiles for the range of $P e$-values considered here.
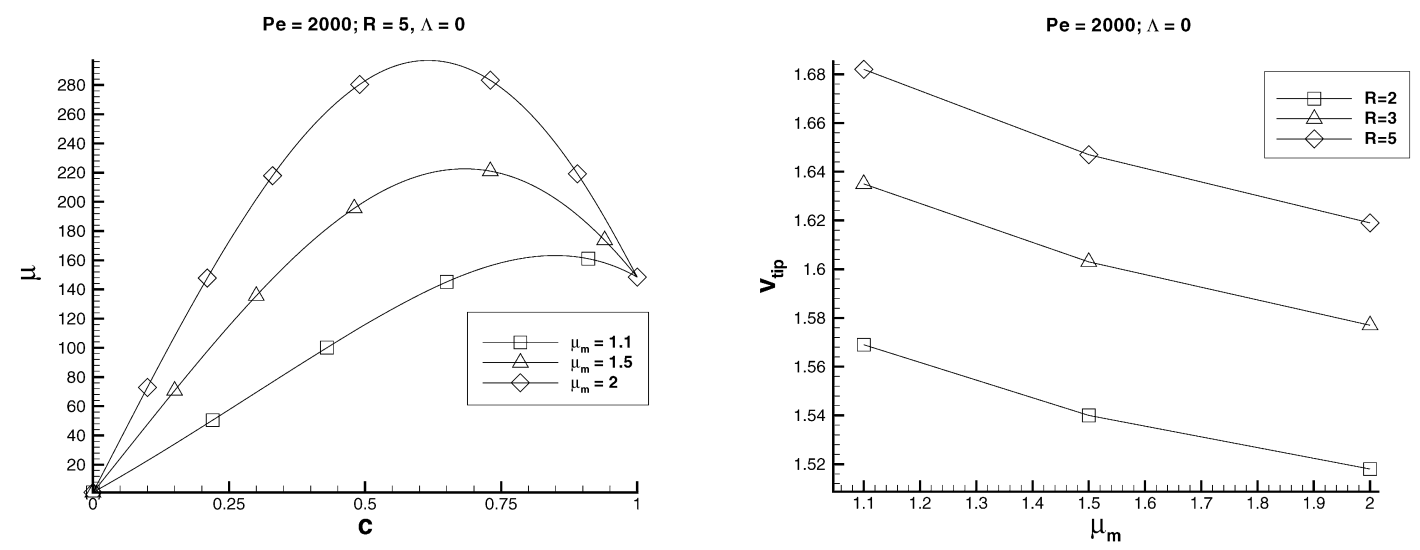

Fig. 5. Left: Viscosity profiles for $R=5$ and different values of $\mu_{m}$. Right: Quasisteady tip velocity as function of $\mu_{m}$ for various viscosity ratios $R$. Similarly to monotonic profiles, the front velocity increases with $R$. As $\mu_{m}$ increases, the overall mobility of the finger is reduced, so that the tip velocity decreases.

a steep concentration layer at the tip of the front cannot be maintained. This is reflected by Fig. 3, which shows the simulation with $P e=2,000$ to reach a quasisteady state, while the front thickness of the $P e=500$ calculation grows diffusively after reaching a minimum. Here the front thickness is defined as the distance between the $c=0.1$ and $c=0.9$ contours at the tip of the finger.

Fig. 4(left) displays the tip velocity $v_{\text {tip }}$ of the quasisteady base state as a function of $P e$ for different values of $R$. The solid lines show the results of a representative nonmonotonic case with $\Lambda=0$ and $\mu_{m}=2$, while the dashed lines depict the monotonic, exponential case for comparison. Note that for the lower values of $P e$ and $R$ the simulations reach a nearly quasisteady state only briefly. We observe that for nonmonotonic profiles the tip velocity is substantially lower than for the corresponding exponential cases with equal $P e$ and $R$. In contrast to the exponential cases, for nonmonotonic profiles the tip velocity increases with $\mathrm{Pe}$ [3]. For both exponential and nonmonotonic profiles, the tip velocity increases with the viscosity contrast.

The front thickness is dominated by the local strain field at the finger tip, which in turn is generated by the difference between the fluid velocity behind the finger tip and the Poiseuille flow ahead of the finger tip. Larger tip velocities generally result in a stronger strain field, and hence in a decreasing front thickness, cf. Fig. 4(right). Interestingly, while the monotonic profiles closely follow the scaling law [13]

$$
d_{0} \sim P e^{-1 / 2},
$$

nonmonotonic viscosity profiles deviate somewhat from this behavior for the range of $P e$-values considered here. 

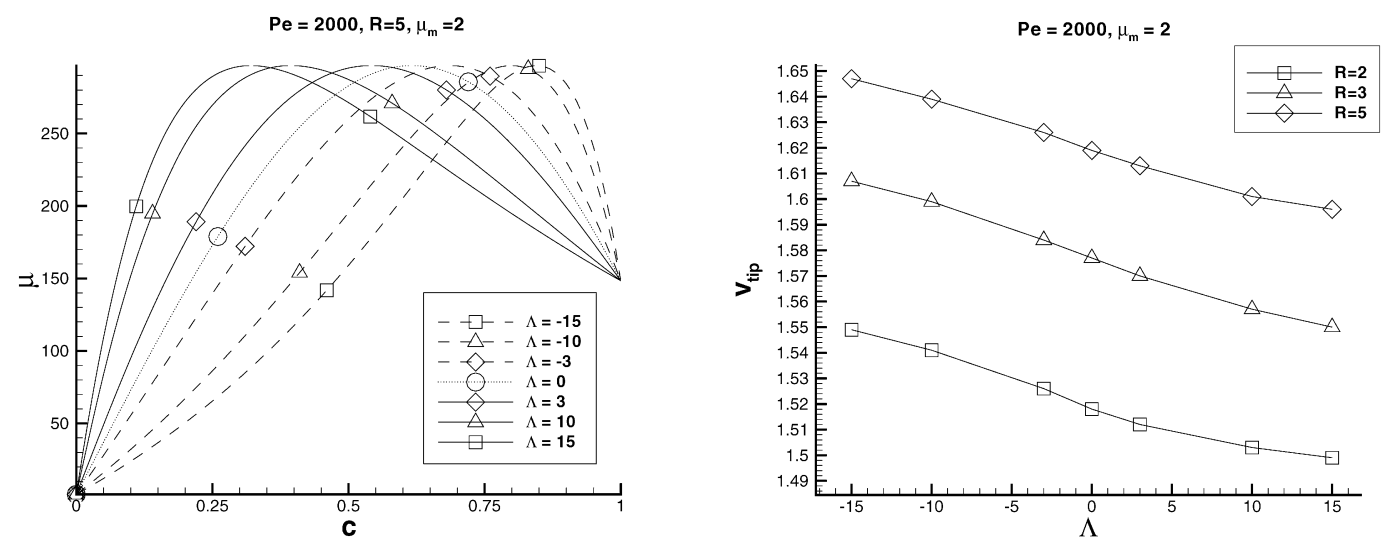

Fig. 6. Left: Viscosity profiles for $R=5$ and various values of $\Lambda$. For larger $\Lambda$, the effective viscosity of the outer layer of the finger increases, which lowers its mobility. Right: Quasisteady tip velocity as a function of $\Lambda$. As a result of the reduced mobility of the finger for increasing values of $\Lambda$, its tip velocity is reduced.

As the maximum $\mu_{m}$ of the viscosity-concentration relationship increases, the diffusive region connecting the finger and the resident fluid experiences an increase in viscosity. This reduces the overall mobility of the finger and thus results in a lower tip velocity, cf. Fig. 5.

Similarly to $\mu_{m}$, the value of the parameter $\Lambda$ affects the viscosity in the diffusive layer connecting the displacing and the resident fluid. Positive values of $\Lambda$ increase the viscosity in this region, thus reducing the finger's mobility and slowing it down, while negative values of $\Lambda$ have the opposite effect, cf. Fig. 6.

\section{Linear stability analysis}

\subsection{Formulation of the eigenvalue problem}

In order to perform the linear stability analysis of the quasisteady displacement, the three-dimensional Stokes equations are linearized around the two-dimensional base state

$$
\begin{aligned}
& u(x, y, z, t)=0+u^{\prime}(x, y, z, t), \\
& v(x, y, z, t)=\bar{v}(y, z)+v^{\prime}(x, y, z, t), \\
& w(x, y, z, t)=\bar{w}(y, z)+w^{\prime}(x, y, z, t), \\
& p(x, y, z, t)=\bar{p}(y, z)+p^{\prime}(x, y, z, t), \\
& c(x, y, z, t)=\bar{c}(y, z)+c^{\prime}(x, y, z, t) .
\end{aligned}
$$

Here, a quantity with the ${ }^{-}$-symbol denotes the base state, while the '-symbol indicates a perturbation quantity. The above expressions are substituted into the governing equations, the base state is subtracted out, and all terms of second or higher order in the perturbations are neglected. The perturbations are assumed to be wavelike in the spanwise $x$-direction

$$
\begin{aligned}
& u^{\prime}(x, y, z, t)=\hat{u}(y, z) \cdot \sin (\beta x) \cdot \mathrm{e}^{\sigma t}, \\
& v^{\prime}(x, y, z, t)=\hat{v}(y, z) \cdot \cos (\beta x) \cdot \mathrm{e}^{\sigma t}, \\
& w^{\prime}(x, y, z, t)=\hat{w}(y, z) \cdot \cos (\beta x) \cdot \mathrm{e}^{\sigma t}, \\
& p^{\prime}(x, y, z, t)=\hat{p}(y, z) \cdot \cos (\beta x) \cdot \mathrm{e}^{\sigma t}, \\
& c^{\prime}(x, y, z, t)=\hat{c}(y, z) \cdot \cos (\beta x) \cdot \mathrm{e}^{\sigma t},
\end{aligned}
$$

where $\sigma$ denotes the growth rate, $\beta$ indicates the wave number, and the ${ }^{\wedge}$-symbol represents the eigenfunctions. We thus obtain a generalized eigenvalue problem of the form

$$
\underline{\underline{A}} \underline{\phi}=\sigma \underline{\underline{B}} \underline{\phi}
$$


with the growth rate $\sigma$ as the eigenvalue. As usual, a positive (negative) eigenvalue indicates unstable (stable) behavior. $\underline{\underline{A}}$ and $\underline{\underline{B}}$ denote the coefficient matrices and are given by

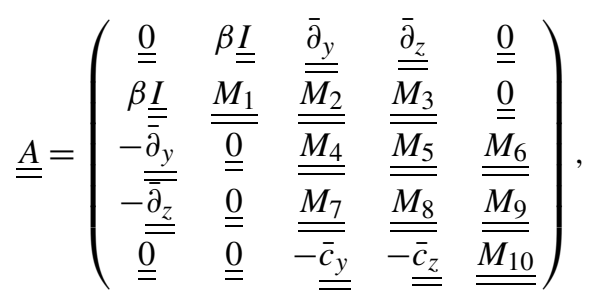

with

$$
\begin{aligned}
& \underline{\underline{M_{1}}}=\bar{\mu}\left(\underline{\underline{\partial_{y y}}}+\underline{\underline{\bar{\partial}_{z z}}}-\beta^{2} \underline{\underline{I}}\right)+\bar{\mu}_{z} \underline{\underline{\bar{\partial}_{z}}}+\bar{\mu}_{y} \underline{\underline{\partial_{y}}}, \\
& \underline{\underline{M_{2}}}=-\beta \bar{\mu}_{y} \underline{\underline{I}} \\
& \underline{\underline{M_{3}}}=-\beta \bar{\mu}_{z} \underline{\underline{I}}, \\
& \underline{\underline{M_{4}}}=2 \bar{\mu}_{y} \underline{\underline{\underline{\partial}}}+\bar{\mu}\left(\underline{\underline{\bar{\partial}_{y y}}}+\underline{\underline{\bar{\partial}_{z z}}}-\beta^{2} \underline{\underline{I}}\right)+\bar{\mu}_{z} \underline{\underline{\bar{\partial}_{z}}}, \\
& \underline{\underline{M_{5}}}=\bar{\mu}_{z} \underline{\underline{\partial_{y}}}, \\
& \underline{\underline{M_{6}}}=2 \bar{v}_{y}\left(\frac{\partial \bar{\mu}_{c}}{\partial y} \underline{\underline{I}}+\bar{\mu}_{c} \underline{\underline{\partial_{y}}}\right)+\left(\bar{v}_{z}+\bar{w}_{y}\right)\left(\frac{\partial \bar{\mu}_{c}}{\partial z} \underline{\underline{I}}+\bar{\mu}_{c} \underline{\underline{\partial}} \bar{\partial}_{z}\right)+\left(\bar{v}_{y y}+\bar{v}_{z z}\right) \bar{\mu}_{c} \underline{\underline{I}}, \\
& \underline{\underline{M_{7}}}=\bar{\mu}_{y} \underline{\underline{\partial_{z}}}, \\
& \underline{\underline{M_{8}}}=2 \bar{\mu}_{z} \underline{\underline{\partial_{z}}}+\bar{\mu}\left(\underline{\underline{\partial_{y y}}}+\underline{\underline{\bar{\partial}_{z z}}}-\beta^{2} \underline{\underline{I}}\right)+\bar{u}_{y} \underline{\underline{\partial_{y}}}, \\
& \underline{\underline{M_{9}}}=2 \bar{w}_{z}\left(\frac{\partial \bar{\mu}_{c}}{\partial z} \underline{\underline{I}}+\bar{\mu}_{c} \underline{\bar{\partial}_{z}}\right)+\left(\bar{v}_{z}+\bar{w}_{y}\right)\left(\frac{\partial \bar{\mu}_{c}}{\partial y} \underline{\underline{I}}+\bar{\mu}_{c} \underline{\underline{\partial}} \underline{=}\right)+\left(\bar{w}_{y y}+\bar{w}_{z z}\right) \bar{\mu}_{c} \underline{\underline{I}}, \\
& \underline{\underline{M_{10}}}=\frac{1}{P e}\left(-\beta^{2} \underline{\underline{I}}+\underline{\underline{\bar{\partial}_{y y}}}+\underline{\underline{\bar{\partial}_{z z}}}\right)-\bar{v} \underline{\underline{\partial_{y}}}-\bar{w} \underline{\underline{\partial_{z}}}
\end{aligned}
$$

and

$$
\underline{\underline{B}}=\left(\begin{array}{ccccc}
\underline{\underline{\underline{0}}} & \underline{\underline{\underline{0}}} & \underline{\underline{\overline{0}}} & \underline{\underline{\underline{0}}} & \underline{\overline{0}} \\
\underline{\overline{0}} & \underline{\overline{0}} & \underline{\overline{0}} & \underline{\overline{0}} & \underline{\overline{0}} \\
\underline{\overline{0}} & \underline{\overline{0}} & \underline{\overline{0}} & \underline{\overline{0}} & \underline{\overline{0}} \\
\underline{\overline{0}} & \underline{\overline{0}} & \underline{\overline{0}} & \underline{\overline{0}} & \underline{\overline{0}} \\
\underline{\underline{0}} & \underline{\underline{0}} & \underline{\underline{0}} & \underline{\underline{0}} & \underline{\underline{I}}
\end{array}\right) \quad \text { and } \quad \phi=\left(\begin{array}{c}
\hat{p} \\
\hat{u} \\
\hat{v} \\
\hat{w} \\
\hat{c}
\end{array}\right) .
$$

The resulting set of equations is discretized on a domain that extends from $z=0$ to $z=0.5$ and is centered around the finger tip in the streamwise direction. This domain has to be taken sufficiently large so that Neumann conditions can be assumed for the perturbations at the upstream and downstream boundaries. At the wall the perturbation velocity and the normal derivative of the concentration perturbation are set to zero, while symmetry boundary conditions are employed at the centerline. The base state velocity and concentration fields, as well as all their required derivatives, are interpolated from the DNS grid to the stability grid using sixth order Lagrangian interpolation. In the $z$-direction the nodes are spaced equidistantly, while a stretched grid is employed on the two subdomains $y \geqslant y_{\text {tip }}$ and $y \leqslant y_{\text {tip }}$ that concentrates the grid points near $y_{\text {tip. }}$. For this purpose a mapping function provided by [14] is employed, cf. [15]. The numerical solution procedure for the generalized eigenvalue problem follows the approach described in detail by [3]. Convergence tests demonstrated that a much higher resolution is necessary for nonmonotonic viscosity profiles, as compared to the monotonic case. For most parameter combinations a resolution of $n_{z}=193$ in the $z$ - and $n_{y}=435$ in the $y$-direction is employed. 


\subsection{Linear stability results}

Fig. 7(left) compares the growth rate $\sigma_{\max }$ of the most dangerous mode as a function of the viscosity ratio $R$ for the nonmonotonic and monotonic profiles. For both cases, the growth rate increases with the viscosity contrast. While the growth rates are similar in magnitude, the nonmonotonic profile generally is slightly less unstable than the monotonic one. The dominant wave number for the nonmonotonic case is somewhat higher than for the exponential counterpart, cf. Fig. 7(right). For those values of $P e$ for which we obtained a quasisteady state, the influence of $P e$ on the growth rate and the dominant wave number was minor (not shown). Taken together, these results show that the influence of the nonmonotonicity on the most amplified wave number and the associated growth rate is relatively minor, and considerably smaller than what was observed by [1] for Darcy flows. A visual inspection of the eigenfunctions shows that they are qualitatively and quantitatively very similar for the monotonic and nonmonotonic profiles (Fig. 8), which in turn reflects the similarity of the base states.

Fig. 9(left) displays the dominant growth rate $\sigma_{\max }$ as a function of the maximum viscosity $\mu_{m}$ for the parameters $P e=2,000$ and $\Lambda=0$. The influence of the maximum of the viscosity profile is seen to be relatively small. As $\mu_{m}$ changes from 1.1 to 2 , the growth rate decreases slightly for large $R$, while there is a mild increase for smaller values of $R$. Similarly, the most dangerous wave number $\beta_{\max }$ does not exhibit a strong dependence on $\mu_{m}$ for $\Lambda=$ const. (Fig. 9(right). In general the dominant wave number decreases slightly as $\mu_{m}$ increases.

[1] had observed a strong influence of the end point gradient parameter $\Lambda$ on the linear stability results for Darcy displacements. Fig. 10 shows the maximum growth rate as a function of $\Lambda$ for different values of $R$. The results are consistent with the Darcy flow by investigation of [1], in that the growth rate shows an overall increase with $\Lambda$.
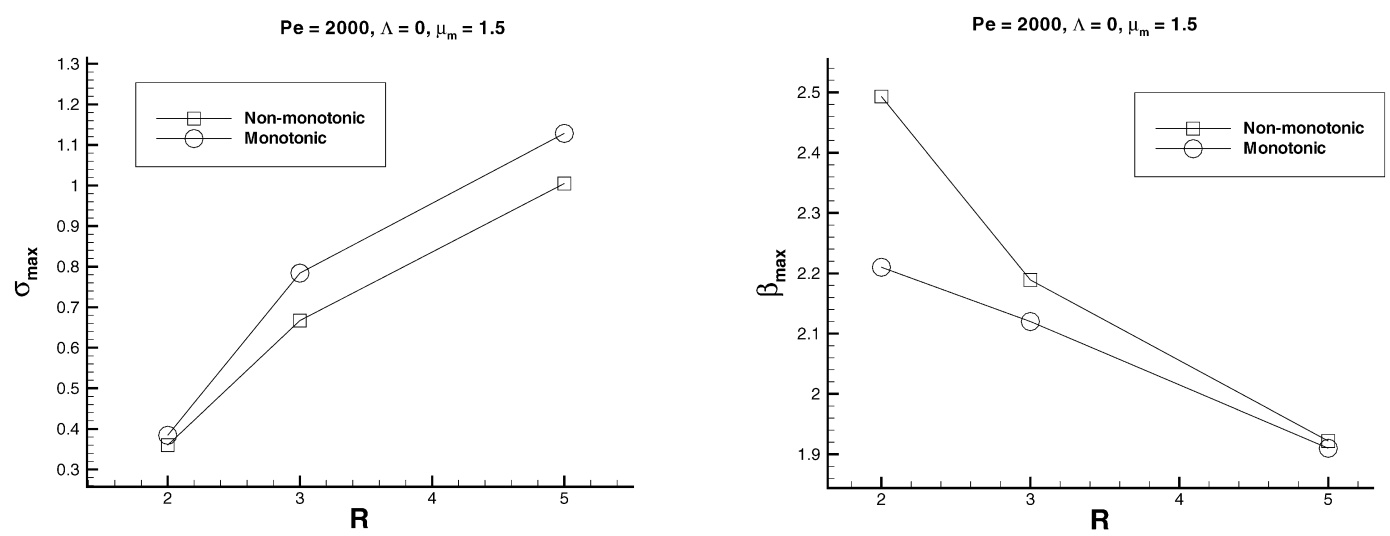

Fig. 7. Comparison of the monotonic and nonmonotonic $\left(\Lambda=0, \mu_{m}=1.5\right)$ viscosity profiles, for different values of $R$ and $P e=2,000$. Left: Maximum growth rate $\sigma_{\max }$. The nonmonotonic profile is found to be slightly less unstable. Right: Most dangerous wave number. The most dangerous wavelength for the nonmonotonic case is slightly lower, as compared to its monotonic counterpart.
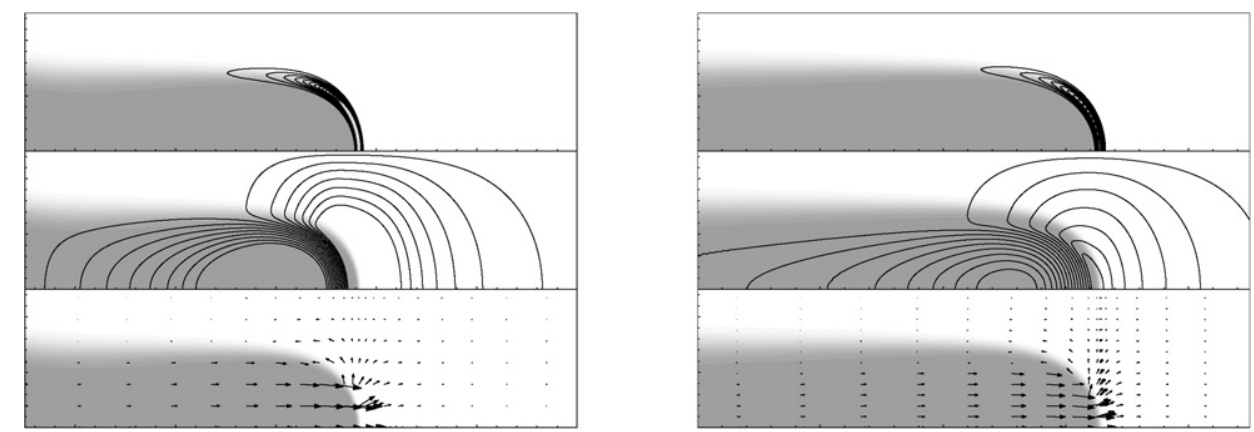

Fig. 8. Comparison of the eigenfunctions for (from top to bottom) $\hat{c}, \hat{u}$, and the combined $\hat{v}$ and $\hat{w}$, for corresponding monotonic (left) and nonmonotonic (right) cases. While there are differences in the detailed shapes of the eigenfunctions, their overall nature remains largely unaffected by the nonmonotonicity of the profiles. 

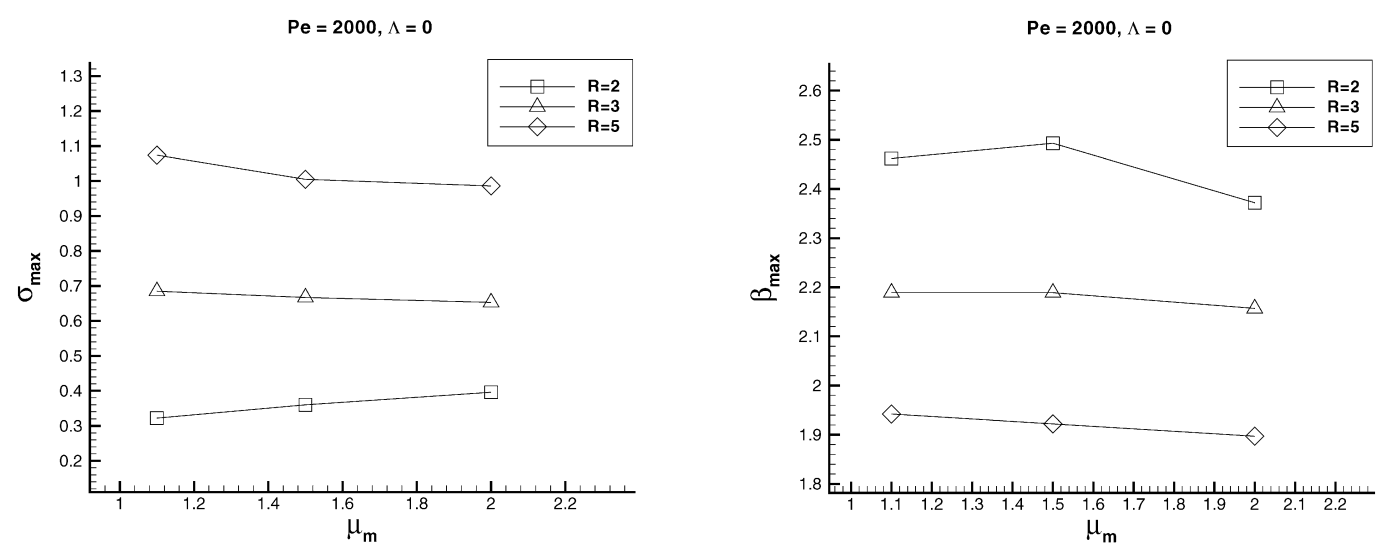

Fig. 9. Left: The dominant growth rate $\sigma_{\max }$ as a function of the viscosity maximum $\mu_{m}$. The variation in the growth rate is relatively small as $\mu_{m}$ increases from 1.1 to 2 . Right: The dominant wave number $\beta_{\max }$ as a function of $\mu_{m}$. As $\mu_{m}$ increases from 1.1 to 2 , the wave number of the most amplified mode is seen to decrease slightly.

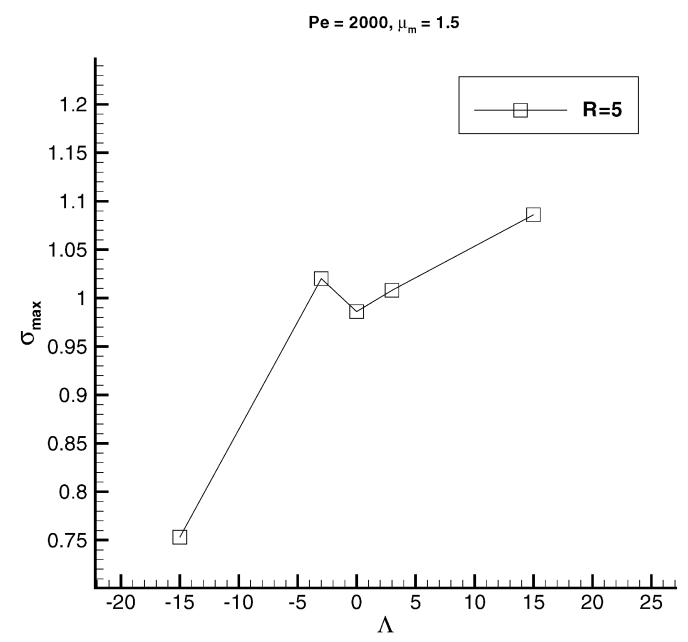

Fig. 10. Growth rate $\sigma_{\max }$ for $\Lambda=[-15,15]$ and $R=3$. The end point gradient parameter is seen to have a substantial effect on the growth rate.

However, [1] had found a substantial influence already at much lower values of $\Lambda$. The wave number $\beta_{\max }$ shows a very weak dependence on $\Lambda$ (not shown).

\section{Discussion and conclusions}

Viscous fingering for neutrally buoyant, miscible fluids in a Hele-Shaw cell has been investigated by means of nonlinear Stokes flow simulations and a subsequent linear stability analysis, with an emphasis on the influence of nonmonotonic viscosity-concentration relationships.

In a first step, quasisteady base states were obtained by means of nonlinear Stokes simulations. Similarly to monotonic profiles, such quasisteady states are seen to develop for large Péclet numbers and viscosity ratios. The tip velocity for nonmonotonic profiles is seen to be somewhat lower than for monotonic cases, which can be explained by the reduced mobility of the finger, as a result of the increased viscosity along its sides. As a result, nonmonotonic profiles have a slightly increased tip thickness. The tip velocity for nonmonotonic profiles is seen to increase slightly with $P e$, in contrast to the exponential profiles. While for monotonic profiles the tip thickness scales with $P e^{-1 / 2}$, this scaling does not hold for nonmonotonic profiles in the range of $P e$-values investigated here. An increase in the maximum viscosity $\mu_{m}$ lowers the overall mobility of the finger, and thus reduces its tip velocity. 
Subsequently, the stability of the above base states was investigated by means of a linear stability analysis. The dominant wave number is seen to be slightly higher for the nonmonotonic case, while the associated growth rate is somewhat smaller. The viscosity maximum $\mu_{m}$ has a fairly small influence on the growth rate and the most amplified wave number. On the other hand, the end point gradient parameter affects the growth rate significantly, but only at relatively large values.

Overall, the nonmonotonicity of the viscosity-concentration relationship is seen to have a much smaller influence on Hele-Shaw displacements than on Darcy flows. The reason for this difference lies in the nature of the base states. For Darcy flows, the base state is characterized by constant velocity and a diffusively decaying concentration (and hence viscosity) profile. This base viscosity profile is strongly affected by the nonmonotonicity. On the other hand, for Hele-Shaw displacements the quasisteady base states are convectively dominated and characterized by sharp fronts. As a result, their shape depends only weakly on the details of the viscosity-concentration relationship. Hence, for Hele-Shaw displacements both the eigenfunctions and the associated growth rates are quite similar for monotonic and nonmonotonic profiles, in contrast to the findings by [1] for Darcy flows.

In this context, it is of interest to mention the work of [16], which takes a different approach towards analyzing similar flows. These authors employ piecewise viscosity functions in order to generate a simplified model for miscible displacements. Depending on which one of the fluids is more viscous, and on whether or not the viscosity profile is monotonic, the authors obtain a hyperbolic or elliptic problem, respectively. This change in type reflects the breakdown of the parallel flow approximation, which cannot capture the structure at the tip. An open question concerns possible links between the change in type and spanwise instability.

\section{Acknowledgements}

Support for this research was received from the NASA Microgravity and NSF/ITR programs, through an NSF equipment grant, and through TeraGrid resources provided by the San Diego Supercomputer Center.

\section{References}

[1] O. Manickam, G.M. Homsy, Stability of miscible displacements in porous media with nonmonotonic viscosity profiles, Phys. Fluids A 5 (1993) 1356-1367.

[2] G.M. Homsy, Viscous fingering in porous media, Annu. Rev. Fluid Mech. 19 (1987) 271.

[3] N. Goyal, E. Meiburg, Miscible displacements in Hele-Shaw cells: Two-dimensional base states and their linear stability, J. Fluid Mech. 558 (2006) 329-355.

[4] C.T. Tan, G.M. Homsy, Stability of miscible displacements in porous media: Rectilinear flow, Phys. Fluids 29 (11) (1986) $3549-3556$.

[5] N. Goyal, H. Pichler, E. Meiburg, Variable density, miscible displacements in a vertical Hele-Shaw cell: linear stability, J. Fluid Mech. (2006), submitted for publication.

[6] E. Lajeunesse, J. Martin, N. Rakotomalala, D. Salin, Y.C. Yortsos, Miscible displacement in a Hele-Shaw cell at high rates, J. Fluid Mech. 398 (1999) 299-319.

[7] O. Manickam, G.M. Homsy, Simulation of viscous fingering in miscible displacements with nonmonotonic viscosity profiles, Phys. Fluids 6 (1994) 95-107.

[8] J.C. Bacri, D. Salin, Y. Yortsos, Analyse lineaire de la stabilite de 1'ecoulement de fluides miscibles en milieus poreux, C. R. Acad. Sci. Paris 314 (1992) 139-144.

[9] C. Pankiewitz, E. Meiburg, Miscible porous media displacements in the quarter five-spot configuration. Part 3. Non-monotonic viscosity profiles, J. Fluid Mech. 388 (1999) 171-195.

[10] A.M. Rogerson, E. Meiburg, Shear stabilization of miscible displacement processes in porous media, Phys. Fluids 17 (5) (2005).

[11] A.M. Rogerson, E. Meiburg, Numerical simulation of miscible displacement processes in porous media flows under gravity, Phys. Fluids 17 (5) (2005).

[12] N. Goyal, E. Meiburg, Unstable density stratification of miscible fluids in a vertical Hele-Shaw cell: Influence of variable viscosity on the linear stability, J. Fluid Mech. 516 (2004) 211-238.

[13] C.Y. Chen, E. Meiburg, Miscible displacements in capillary tubes. Part 2. Numerical simulations, J. Fluid Mech. 326 (1996) 57-90.

[14] C.A.J. Fletcher, Computational Techniques for Fluid Dynamics, vol. 2, Springer-Verlag, New York, 1990.

[15] S.H. Vanaparthy, E. Meiburg, D. Wilhelm, Density-driven instabilities of miscible fluids in a capillary tube: Linear stability analysis, J. Fluid Mech. 497 (2003) 99-121.

[16] M. Shariati, L. Talon, J. Martin, N. Rakotomalala, D. Salin, Y.C. Yortsos, Fluid displacement between two parallel plates: a non-empirical model displaying change of type from hyperbolic to elliptic equations, J. Fluid Mech. 516 (2004) 211-238. 